\title{
Large-Eddy Simulation of Transition to Turbulence in Boundary Layers ${ }^{1}$
}

\author{
Xiaoli Huai, \\ Department of Mechanical Engineering, \\ University of Maryland, College Park, MD 20742, U.S.A. \\ Ronald D. Joslin, \\ Flow Modeling and Control Branch, \\ NASA Langley Research Center, Hampton, VA 23681, U.S.A. \\ and Ugo Piomelli \\ Department of Mechanical Engineering, \\ University of Maryland, College Park, MD 20742, U.S.A.
}

\begin{abstract}
Large-eddy simulation results for laminar-to-turbulent transition in a spatially developing boundary layer are presented. The disturbances are ingested into a laminar flow through an unsteady suction-and-blowing strip. The filtered, three-dimensional timedependent Navier-Stokes equations are integrated numerically using spectral, high-order finite-difference, and three-stage low-storage Runge-Kutta methods. The buffer-domain technique is used for the outflow boundary condition. The localized dynamic model used to parameterize the subgrid-scale stresses begins to have a significant impact at the beginning of the nonlinear transition (or intermittency) region. The flow structures commonly found in experiments are also observed in the present simulation; the computed linear instability modes and secondary instability lambda-vortex structures are in agreement with the experiments, and the streak-like-structures and turbulent statistics compare with both the experiments and the theory. The physics captured in the present LES are consistent with the experiments and the full Navier-Stokes simulation (DNS), at a significant fraction of the DNS cost. A comparison of the results obtained with several SGS models shows that the localized model gives accurate results both in a statistical sense and in terms of predicting the dynamics of the energy-carrying eddies, without ad hoc adjustments.
\end{abstract}

\section{Introduction}

The problem of transition from laminar to turbulent flow in boundary layers is of great practical interest. Transition studies are motivated by a need to understand this physical process and to apply this knowledge to the prediction and control of transition. For example, the low skin-friction drag of laminar boundary layer flow compared with turbulent flow is very attractive to those who design high performance automobiles and aircrafts. On the

\footnotetext{
${ }^{1}$ This research was supported by NASA under grant No. NAG-1-1089. Computer resources were provided by the NASA Langley Research Center and NASA Ames Research Center.
} 
other hand, there are many cases where the high mixing and heat-transfer rates of turbulent boundary-layer are desirable, e.g., for combustion.

Theoretical and experimental work to date in transition studies was reviewed in the recent paper by Kachanov (1994). It is well known today that natural transition due to the amplification of small initial disturbances proceeds through a sequence of stages. The first stage is the two-dimensional (2-D) development of slowly growing (on viscous time-scales) TollmienSchlichting (TS) waves, which can be predicted accurately by the linear stability theory. The rapid growth (on convective time-scales) of infinitesimal three-dimensional disturbances on the base of finite-amplitude 2-D waves, which is explained by secondary instability theory, constitutes the second stage, and is followed by the appearance of small-scale motion and the final stages of transition. Herbert (1988) distinguished three types of secondary instability modes: fundamental, subharmonic and detuned. The fundamental and subharmonic instabilities lead respectively to aligned and staggered patterns of $\Lambda$-vortices observed in controlled experiments of transition. The detuned type leads to a combination pattern in which the $\Lambda$-vortices alternate between aligned and staggered patterns. If the initial disturbances are strong enough (for free-stream turbulence levels above 0.5\%, Roach and Brierley 1992; Voke and Yang 1995), however, the transition will go into the nonlinear interaction stage directly, and the linear instability stage is bypassed; the transition is then called bypass transition. The present study focuses only on natural transition.

It is now readily accepted that the initial stages, including receptivity and the linear and weakly nonlinear instability amplification, can be predicted quite accurately for many receptivity mechanisms or instabilities. What remains a significant challenge even with today's supercomputers is the understanding and ability to compute and predict accurately the nonlinear intermittency transition region. This ability is key for design because the maximum skin friction and temperature peaks occur in this region. Also, this region entails the presence of a great variety of scales to resolve. Hence, if a computational tool were available that could capture the relevant physics of the flow with only a modest cost penalty, it would provide the means to study the flow physics and validate proposed theories to model this complex region.

Progress have also been made in the simulation capabilities of transition studies. Many of the previous numerical simulations were summarized in the review paper by Kleiser and Zang (1991). Although it is natural to simulate transition with a spatial approach, because transition in boundary-layer flows evolves in the streamwise direction, the majority of numerical simulations have involved computing the temporal growth of the instabilities. Spatial simulations of transition have been limited mainly by the extreme computational resolution requirements in the spatially-growing (streamwise) direction, and also by the problems associated with assigning the proper inflow and outflow boundary conditions. It has been observed in experiments that the whole process of transition can require up to 25 wavelengths of the primary TS waves. Furthermore, the late stages of transition involve length scales between one and two orders of magnitude smaller than the TS scale. Thus, providing enough grid points to resolve this phenomena in such a long region is a daunting prospect. By contrast, in temporal simulations only one or two wavelengths need to be resolved, owing to the assumption of streamwise periodicity, and the assumption of parallel mean flow is generally made.

Among the researchers that carried out spatial simulations of transition by direct simulations, Murdock (1986) simulated the K-type transition for the conditions of the Klebanoff et al. (1962) experiment, Fasel et al. (1990) simulated the early three-dimensional stages of both fundamental and subharmonic transition. Recently, Bestek and coworkers (1994) calculated the 2D boundary layer transition under strong adverse pressure gradient, and Lundbladh et al. (1994) carried out the direct numerical simulation of bypass transition in both channel and flat plate boundary layer flows. Joslin and Streett (1994) and Joslin (1995) have 
simulated linear and nonlinear stationary crossflow vortex instability growth in swept wedge boundary-layer flows. Finally, Joslin (1995) used a fully 3D (non-periodic) DNS approach to simulate the evolution of instabilities in attachment-line flow.

Large-eddy simulation (LES) is an alternative approach for the numerical solution of turbulent and transitional flow problems. Unlike the direct numerical simulation (DNS) in which all scales of motion are resolved, in LES only the dynamically important (large) scales are resolved, which results in significant decrease of the CPU time required for a simulation. The effect of the unresolved small scales on the large scales is modeled. Since the small scales tend to be more homogeneous and isotropic, the modelling can be simpler and more universal, compared with that in the Reynolds-averaged approach.

While the application of LES to turbulent flows dates back to the seventies, only recently has this technique been used to the study of transitional flow. Piomelli et al. (1990), Piomelli and Zang (1991) and Germano et al. (1991) computed the transition in temporally developing boundary layer and plane channel flow. Their results indicate that, at the early stages of transition, the eddy viscosity must be inactive to allow the correct growth of the perturbations. The dynamic model (Germano et al. 1991) achieves this result without the ad hoc corrections required by other models. Voke and Yang (1995) performed large-eddy simulation of bypass transition in a flat plate boundary layer, using a low-Reynolds-number correction for the Smagorinsky (1963) model; the properties of the simulated transition match those found experimentally. Recently, Ducros et al. (1996) have performed an LES calculation of transition in a mildly compressible boundary layer using the Filtered Structure Function model. They obtain results that follow the expected trends in the early part of the transition region, but present no experimental or theoretical data to demonstrate quantitative agreement. In the late stages of transition and in turbulence, their resolution is too coarse, and the results, in the late breakdown stages and beyond, do not approach the expected turbulent laws.

Large-eddy simulation, by definition, is a technique in which not all scales of motion are resolved. One question that may arise when applying it to transition problems regards the capability of LES to predict the development of shear layers and vortices whose scale is close the numerical filter. A subgrid-scale (SGS) model suitable for LES applications should not dissipate the energy of the low level perturbations during the initial stages of transition, but should reproduce the energy transfer to the unresolved scales during the nonlinear stages when such small, marginally resolved structures, are generated.

Previous work on the application of LES to transitional flows concentrated on the prediction of statistical data (mean flow, Reynolds stresses, etc.) and only passing mention was devoted to the effect of the SGS model on the development of the vortical structures. This paper, on the other hand, will emphasize this issue, to illustrate how a localized eddy viscosity model responds to the local characteristics of the flow in such a way as to predict accurately the development of such structures, and compare this behavior with that of other models. In this work, we intend to simulate accurately the entire transition process, including the laminar, transitional and turbulent flow regimes.

The numerical method will be introduced first, followed by the simulation description; the results will then be presented along with some comparisons with existing experimental and numerical data; the issue of the prediction of the vortical structures, and the effect of the eddy viscosity on the simulation will then be discussed. Finally, conclusions will be presented. 


\section{Problem formulation}

\section{Mathematical formulation}

The present technique for simulating transitional flow relies on the decomposition of the instantaneous velocity $\tilde{u}_{i}(\mathbf{x}, t)$ and pressure $\tilde{p}(\mathbf{x}, t)$ into base, $U_{o i}(\mathbf{x})$ and $P_{o}(\mathbf{x})$, and disturbance, $u_{i}(\mathbf{x}, t)$ and $p(\mathbf{x}, t)$, components as

$$
\tilde{u}_{i}(\mathbf{x}, t)=U_{o i}(\mathbf{x})+u_{i}(\mathbf{x}, t) \text { and } \tilde{p}(\mathbf{x}, t)=P_{o}(\mathbf{x})+p(\mathbf{x}, t),
$$

where $\mathbf{x}=(x, y, z)$ are the streamwise, wall-normal, and spanwise coordinates and $t$ is time. The base flow is the corresponding streamwise growing laminar flow field and is given by the Blasius similarity solutions.

After substituting (1) into the Navier-Stokes equations and subtracting out the base-flow equations, the disturbance equations result and are given as

$$
\frac{\partial u_{i}}{\partial t}+u_{j} \frac{\partial u_{i}}{\partial x_{j}}+U_{o j} \frac{\partial u_{i}}{\partial x_{j}}+u_{j} \frac{\partial U_{o i}}{\partial x_{j}}=-\frac{\partial p}{\partial x_{i}}+\frac{1}{R e} \frac{\partial^{2} u_{i}}{\partial x_{j} \partial x_{j}}
$$

and

$$
\frac{\partial u_{i}}{\partial x_{i}}=0
$$

where the Reynolds number $R e=U_{\infty} \delta^{*} / \nu, U_{\infty}$ is the freestream velocity and $\delta^{*}$ is the boundary-layer displacement thickness at a reference streamwise location. The disturbance velocity boundary conditions are $u_{i}(\mathbf{x}, t)=0$ at $y=0$ and $y \rightarrow \infty$.

In LES, the large-scale (grid-resolved) components of the velocity and pressure are calculated and the effects of the small, unresolved scales are modeled. By applying the filtering operation

$$
\bar{f}(\mathbf{x})=\int_{D} f\left(\mathbf{x}^{\prime}\right) G\left(\mathbf{x}, \mathbf{x}^{\prime}\right) d \mathbf{x}^{\prime}
$$

(where $G$ is the filter function and $D$ is the entire domain) to (2) and (3), the governing equations for the large-scale velocity and pressure can be obtained:

$$
\frac{\partial \bar{u}_{i}}{\partial t}+\bar{u}_{j} \frac{\partial \bar{u}_{i}}{\partial x_{j}}+U_{o j} \frac{\partial \bar{u}_{i}}{\partial x_{j}}+\bar{u}_{j} \frac{\partial U_{o i}}{\partial x_{j}}=-\frac{\partial \bar{p}}{\partial x_{i}}-\frac{\partial \tau_{i j}}{\partial x_{j}}+\frac{1}{R e} \frac{\partial^{2} \bar{u}_{i}}{\partial x_{j} \partial x_{j}}
$$

and

$$
\frac{\partial \bar{u}_{i}}{\partial x_{i}}=0
$$

where $\tau_{i j}$ is the subgrid-scale (SGS) stress tensor given by $\tau_{i j}=\overline{u_{i} u_{j}}-\bar{u}_{i} \bar{u}_{j}$, which must be modeled.

Here, $\tau_{i j}$ is modeled by a localized version (Piomelli and Liu, 1995) of the dynamic SGS model (Germano et al. 1991). Local five point averaging in the streamwise and spanwise and three point averaging in the wall-normal direction is used in calculating the model coefficient. The total viscosity (molecular + eddy viscosity) is also forced to be non-negative to ensure numerical stability.

\section{Numerical method}

The filtered Navier-Stokes equations are solved using the fractional time step method (Chorin, 1968). Fourth-order finite difference and fourth-order compact difference schemes 

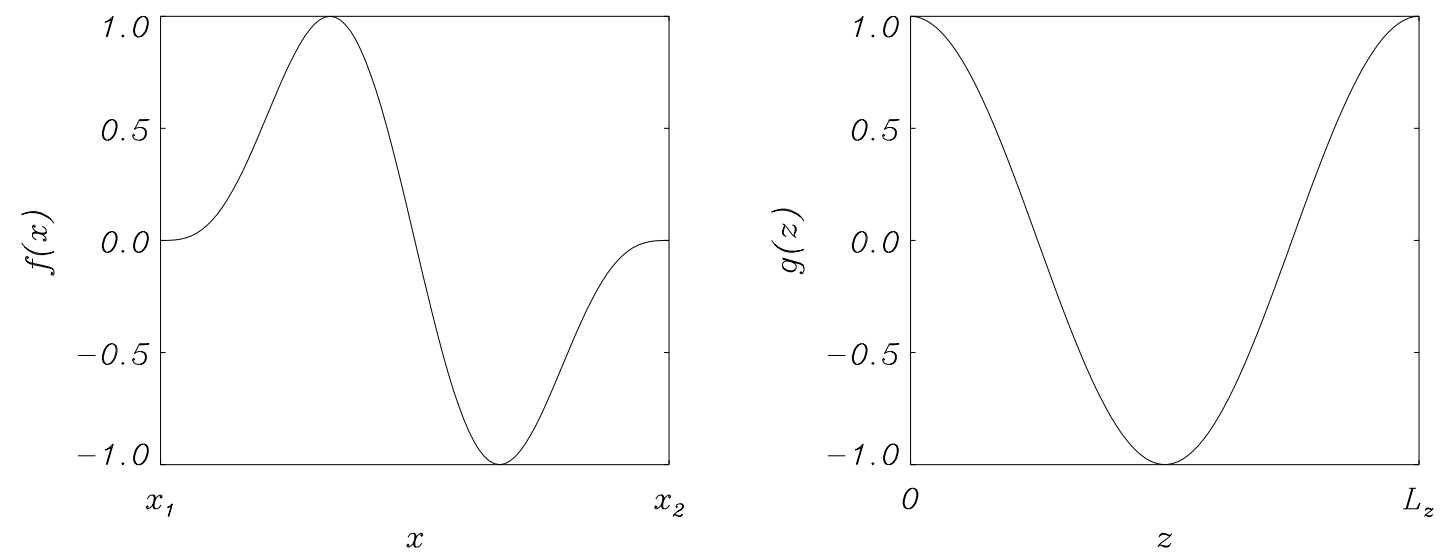

Figure 1: Modulation functions for suction/blowing.

are used in the streamwise direction $(x)$ for the pressure and the momentum equations, respectively, Chebychev series are used in the wall-normal direction $(y)$, and Fourier series in the spanwise direction $(z)$. Implicit Crank-Nicolson time-advancement is used for the wallnormal diffusion terms and a three-stage Runge-Kutta scheme for the remaining terms. A code validation study was previously performed by Joslin et al. $(1992,1993)$.

One of the major difficulties associated with the numerical simulation of spatially developing boundary-layer transition is to define the outflow boundary conditions. Here the buffer domain technique proposed by Streett and Macaraeg (1989) is used, in which the governing equations are gradually parabolized in a buffer region that is appended to the end of the computational domain, thus eliminating the necessity of applying outflow boundary conditions.

\section{Test-case parameters}

In the present simulation, the undisturbed laminar boundary layer is the base flow. Small disturbances are introduced into the flow through a suction/blowing strip at an upstream location. Since these disturbances are unstable, they are amplified as they propagate downstream, and the flow goes through laminar, transition and turbulent stages consecutively.

At the suction/blowing strip, a non-zero normal velocity $v$ is applied. Two frequences are introduced into the flow by

$$
v(x, z, t)=A_{1} f(x) \sin (\omega t)+A_{1 / 2} f(x) g(z) \sin \left(\frac{\omega}{2} t+\phi\right)
$$

where $A_{1}$ and $A_{1 / 2}$ are the disturbance amplitudes, $\phi$ is the phase shift between these two modes, and $f(x), g(z)$ are the modulation functions shown in Fig. 1. The disturbance parameters are chosen to match the experiment of Kachanov and Levchenko (1984) for controlled subharmonic breakdown and are given in Table 1.

To simulate transition in a spatially developing boundary layer, one must use a computational domain that is long enough in the streamwise direction so that laminar, transition and turbulent stages can all be observed, and the grid resolution must be fine enough to ensure accuracy. Two computational boxes were used for the present simulation (see Fig. 2). The first box covers the laminar region, the second one the transition and turbulent regions. In the first box, a fairly coarse grid can be used. In the second box, however, the grid resolution has to be increased substantially to resolve the small scales present in the later stage 


\begin{tabular}{||c||c||c||}
\hline & fundamental mode & subharmonic mode \\
\hline frequency & $\omega=0.0916$ & $\omega / 2$ \\
\hline streamwise wavenumber & $\alpha=0.245$ & \\
\hline streamwise wavelength & $\lambda_{x}=25.6$ & $\beta=0.249$ \\
\hline spanwise wavenumber & & $\lambda_{z}=25.3$ \\
\hline spanwise wavelength & & $A_{\frac{1}{2}}=10^{-5}$ \\
\hline maximum amplitude & $A_{1}=10^{-3}$ & \multicolumn{2}{||||}{$\phi=0$} \\
\hline \hline phase shift & \multicolumn{2}{|c||}{740} \\
\hline reference $R e$ & \multicolumn{2}{|c||}{605} \\
\hline inflow $R e$ & \multicolumn{2}{|c||}{}
\end{tabular}

Table 1: Parameters for the simulation of subharmonic breakdown

\begin{tabular}{||c||c||}
\hline Box I & Box II \\
\hline$L_{x}=487, L_{y}=50, L_{z}=25$ & $L_{x}=359, L_{y}=100, L_{z}=25$ \\
\hline$n x=571, n y=41, n z=9$ & $n x=561, n y=65, n z=33$ \\
\hline$\Delta x=0.86, \Delta y=0.0085, \Delta z=3.2$ & $\Delta x=0.64, \Delta y=0.006, \Delta z=0.79$ \\
\hline & $\Delta x^{+}=21, \Delta y^{+}=0.2, \Delta z^{+}=26$ \\
\hline
\end{tabular}

Table 2: Dimensions of the computational boxes

of transition. In both boxes, the grid points are evenly distributed in both streamwise $(x)$ and spanwise $(z)$ directions. In the wall-normal direction, the grid points are clustered in the near wall region. The box sizes, the grid sizes and the number of grid points used in the simulation are shown in Table 2, where $\Delta y$ is the first wall normal grid size, i.e., the distance from the wall to the first grid point. This grid is finer by a factor of two in each direction than that used by Ducros et al. (1996).

A buffer region with a streamwise length of $3 \lambda_{x}$ is located at the end of each computational box for the numerical outflow boundary conditions. Numerical experiments indicate that this length is sufficient to allow the results in the useful region to be unaffected by the buffer region. The total lengths of the two computational domains are $19 \lambda_{x}$ and $14 \lambda_{x}$ respectively. Both boxes have the same spanwise length $\lambda_{z}$.

The suction/blowing strip is located $2 \lambda_{x}$ downstream of the inlet and spans $\lambda_{x}$ and $\lambda_{z}$ in the streamwise and spanwise directions respectively. At the inflow of the first box, the disturbance velocity components $(u, v, w)$ are equal to zero. This is a valid assumption for the inflow boundary conditions because the suction/blowing strip is sufficiently far from the inflow. At the end of the first useful region $\left(x=16 \lambda_{x}\right)$, the velocity data are saved every time step for two periods of the fundamental wave, i.e., one period of the subharmonic wave. This information is used for the inflow boundary conditions for the second box. By using two periods of inflow data, no useful information is lost since waves with frequencies lower than that of the subharmonic mode are still not present in the flow up to this location. Numerical experiments indicate that, as long as the interface between the domains is located before the strongly nonlinear region, no significant disturbance is introduced by the interpolations required by this procedure. 


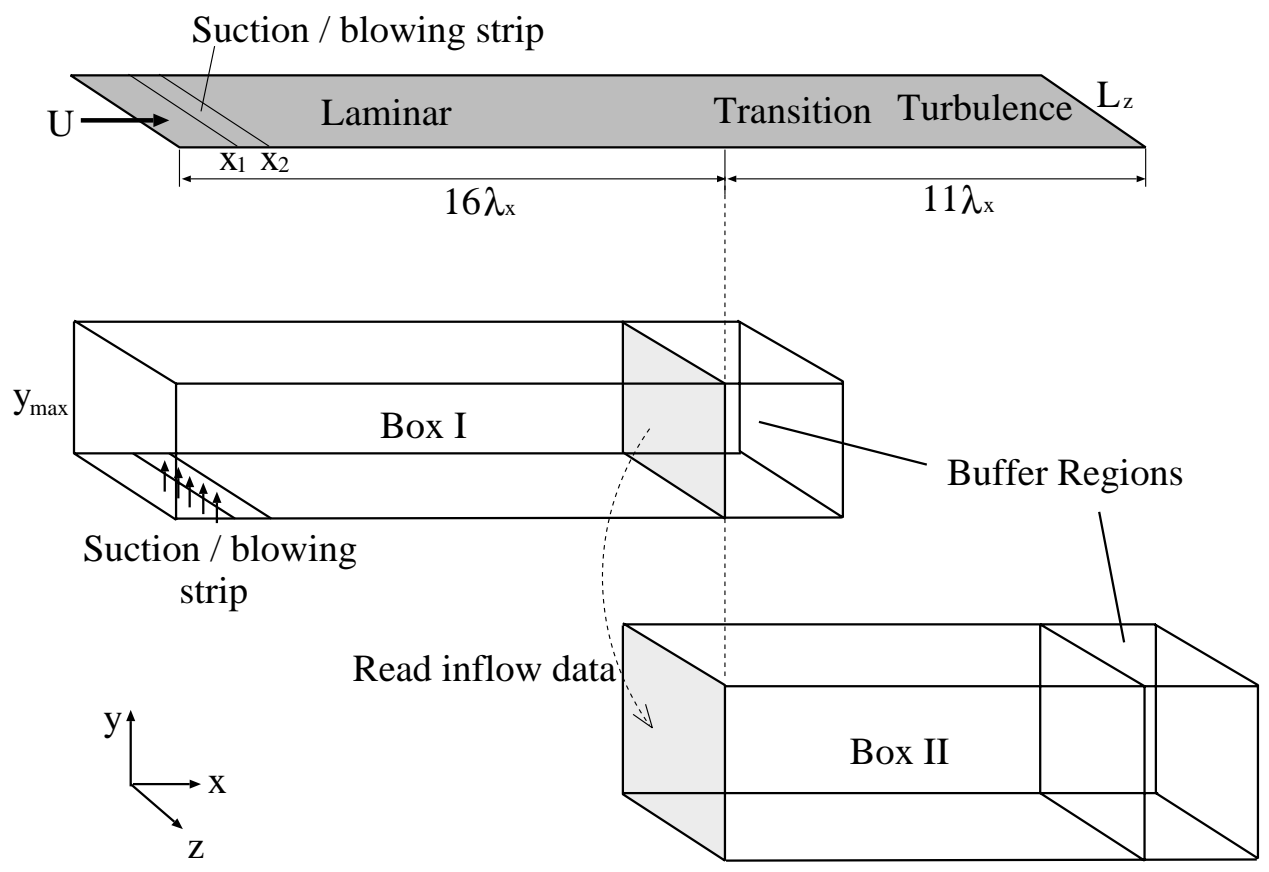

Figure 2: Coordinate system and computational boxes.

\section{Simulation results}

In this section, the numerical results in the two useful regions of the computational boxes will be presented. In the first box, weakly nonlinear growth of the disturbances is observed. In the second box, the flow goes through laminar breakdown and the final transition to turbulence. Numerical results will first be shown, along with some comparisons with experimental and DNS data whenever possible, to validate the present results. The effects of the eddy viscosity on the resolved structures, as well as the intermittency model, will then be presented.

\section{Linear and weakly nonlinear stages}

In the first computational box, the fundamental wave initially grows before it reaches the second branch of the neutral curve, and the subharmonic modes grow much faster due to the secondary instability. Figure 3 shows the streamwise evolution of amplitude and phase of several harmonics for the streamwise velocity $u$, compared with the experiment by Kachanov and Levchenko (1984), where the Reynolds number is defined as $R e_{x}=x U_{\infty} / \nu$ and $x$ is the distance from the leading edge of the plate. Good overall agreement is obtained between the numerical simulation and the experiment. Towards the end of the computational domain, the simulation tends to give higher amplitudes for all the frequency modes shown here. In the experiment the fundamental mode $(\omega)$ and its harmonics $(2 \omega$ and $3 \omega)$ first saturate and then decrease, whereas in the simulation, their amplitude never decreases even though saturation can be seen. The same phenomena was observed in the direct numerical simulation of Fasel et al. (1990), who attributed it to insufficient resolution in the spanwise direction in their simulation, because only the first and second harmonic components in $z$ were included in the computation. However, in the present simulation the same trends are observed although the leading terms of nonlinear interactions with high harmonic components are included. For the 

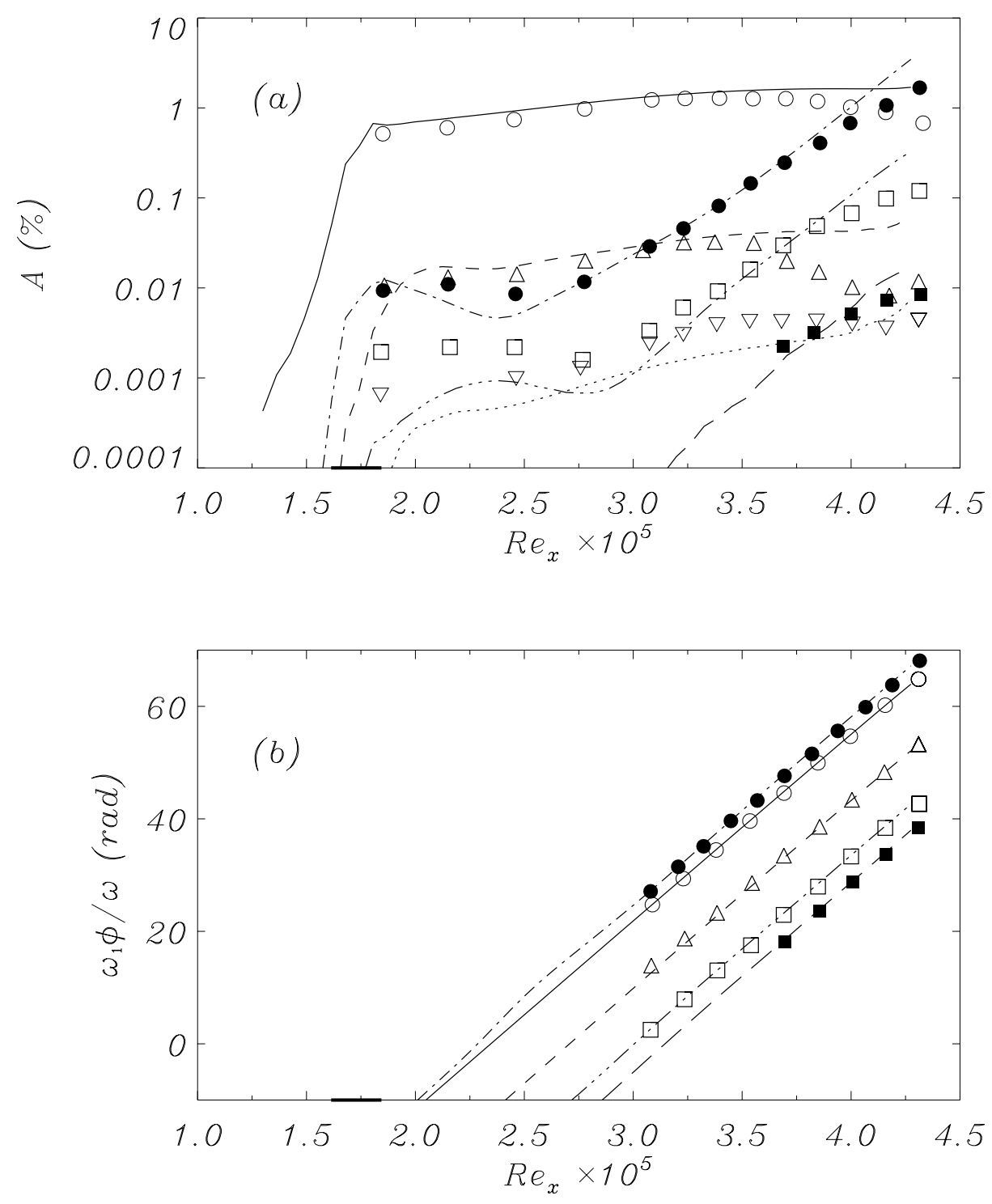

Figure 3: Streamwise evolution of amplitude (a) and phase (b) of selected harmonics at $y / \delta=$ $0.26, z=0$ in the first computational box. Lines: LES; symbols: experiment (Kachanov and Levchenko, 1984). — $○: \omega ;--, \bullet: \omega / 2 ;---, \triangle: 2 \omega ;-\cdots-, \square: 3 \omega / 2 ;---, \mathbf{\square}:$ $5 \omega / 2 ; \cdots \cdots \cdots, \nabla 3 \omega$. 
same test case, Joslin et al. (1993) resolved the instabilities and raised the inconsistency issue between previous theoretical/computational comparisons and the Kachanov and Levchenko (1984) experiments. Imposing a nonzero pressure gradient on a variation in the recorded fundamental frequency, they showed much better agreement in the computed and experimental fundamental modes; however, the subharmonic modes were in worse agreement. Their results suggest that there was insufficient information about the initial spectrum in the experiments to simulate adequately the same breakdown with computations. This could be due to the fact that in the simulation only two frequency modes are introduced into the flow, and nonlinear wave interactions only occur between the fundamental mode, the subharmonic mode and other harmonics generated by nonlinear wave interactions, whereas in the experiment a broad spectrum of low-frequency modes, besides the subharmonic, was amplified to a significant level from background noise, due to resonant interactions with the fundamental mode. Because of the existence and amplification of the low-frequency spectrum, wave interactions would then be much more complicated in the experiment, and transition in the experiment and in the LES may go through different routes; this is supported by the observation that the later stages of transition in the simulation feature high shear layer breakdown, whereas the experiment featured fast spectrum filling without spikes. It is also interesting to note that the fundamental wave and its harmonics tail up at the end of the useful region, which indicates the rapid joint growth of both fundamental and subharmonic waves at late stages of subharmonic breakdown after crossing their amplification curves, as was found in both experimental and theoretical studies (Maslennikova and Zelman, 1985; Crouch and Herbert, 1993).

The comparison of the streamwise phase evolution (Fig. 3b) shows excellent agreement between the simulation and the experimental data. All the modes have the same constant downstream propagation velocity throughout the whole box. This wave synchronism condition, which should be satisfied in order to have resonant wave interactions between all these modes, was observed in the experiments of Corke and Mangano (1989) and Kachanov and Levchenko (1984).

Wall-normal distributions of amplitude and phase of the fundamental and subharmonic modes for the velocity $u$ are shown in Fig. 4. The comparison with the experiment is also good.

\section{Nonlinear transition stage and turbulent region}

In the second box, the disturbances grow further, all the frequency modes interact in a more complex manner, and the flow goes through laminar breakdown and final transition to turbulence. Fig. 5 shows the mean streamwise velocity in the wall-normal direction at five equally spaced streamwise locations, with the first one at the beginning and the last one at the end of the computational domain. The mean quantities shown in this section were obtained by averaging over both the spanwise direction and time, using 60 instantenous flow fields spanning $3 T$ in time (where $T$ is the period of the fundamental wave) taken after a steady state was reached. As the flow evolves in the streamwise direction, the mean velocity changes from a laminar profile to a turbulent one. At the last location the velocity is in very good agreement with the DNS data of Spalart (1988), although the friction velocity for the LES is smaller than that for the DNS by $3 \%$, due to the fact that the Reynolds number at this location is $R e_{\delta^{*}}=1372$, higher than that of the DNS, $R e_{\delta^{*}}=1000$. Figure 6 gives the rms of all three velocity components normalized by the friction velocity at the last streamwise location. Good agreement with DNS is again achieved.

Figure 7 shows the streamwise variation of shape factor obtained from the large-eddy simulation. The shape factor in the turbulent region is slightly lower than that of the DNS, again due to Reynolds number difference. 

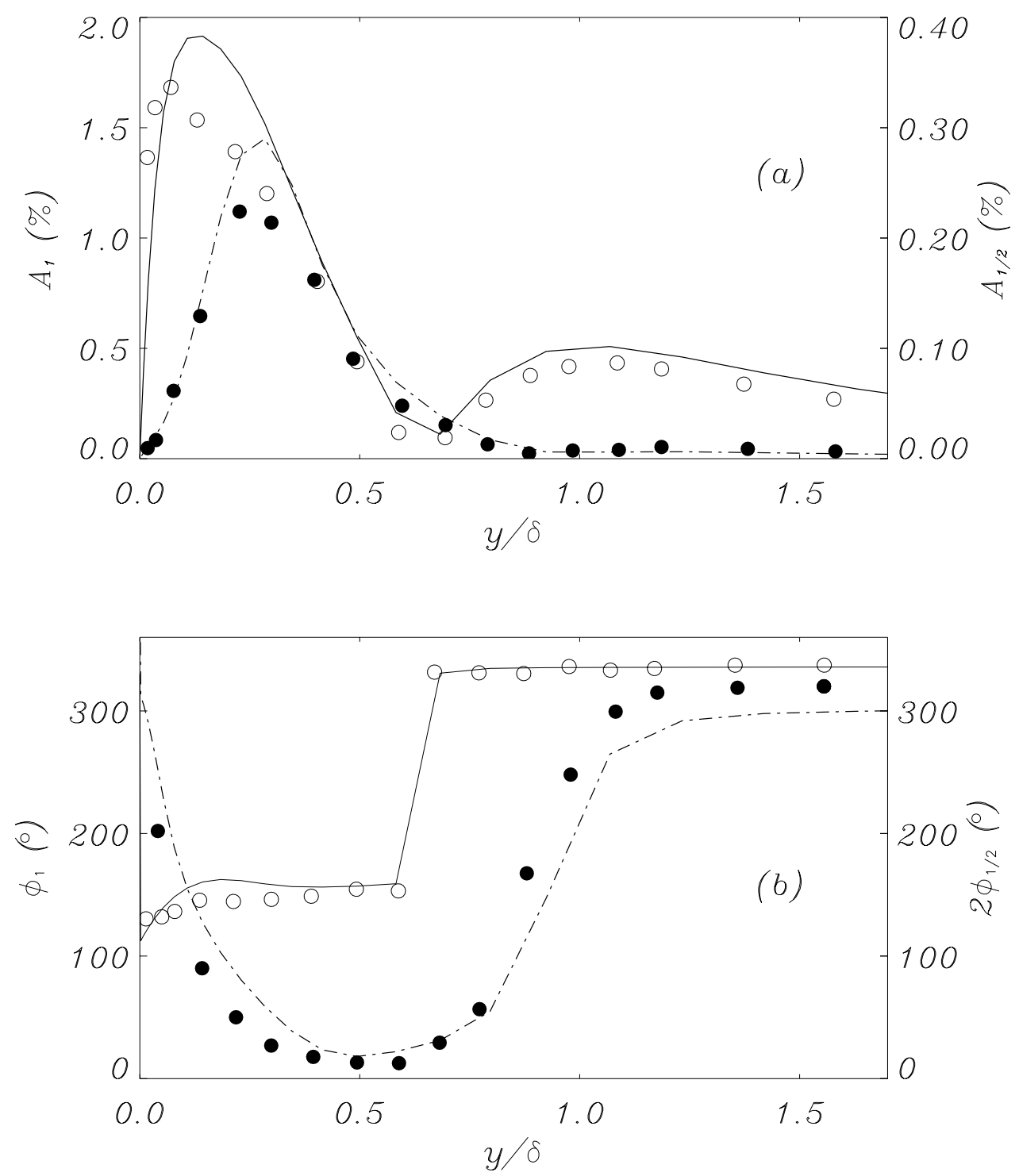

Figure 4: Wall-normal distribution of amplitude (a) and phase (b) of the fundamental and subharmonic waves in the $z=0$ plane, $R e_{x}=3.6 \times 10^{5}$. Lines: LES; symbols: experiment (Kachanov and Levchenko, 1984). - , ० : $\omega ;--\cdots, \bullet \omega / 2$. 


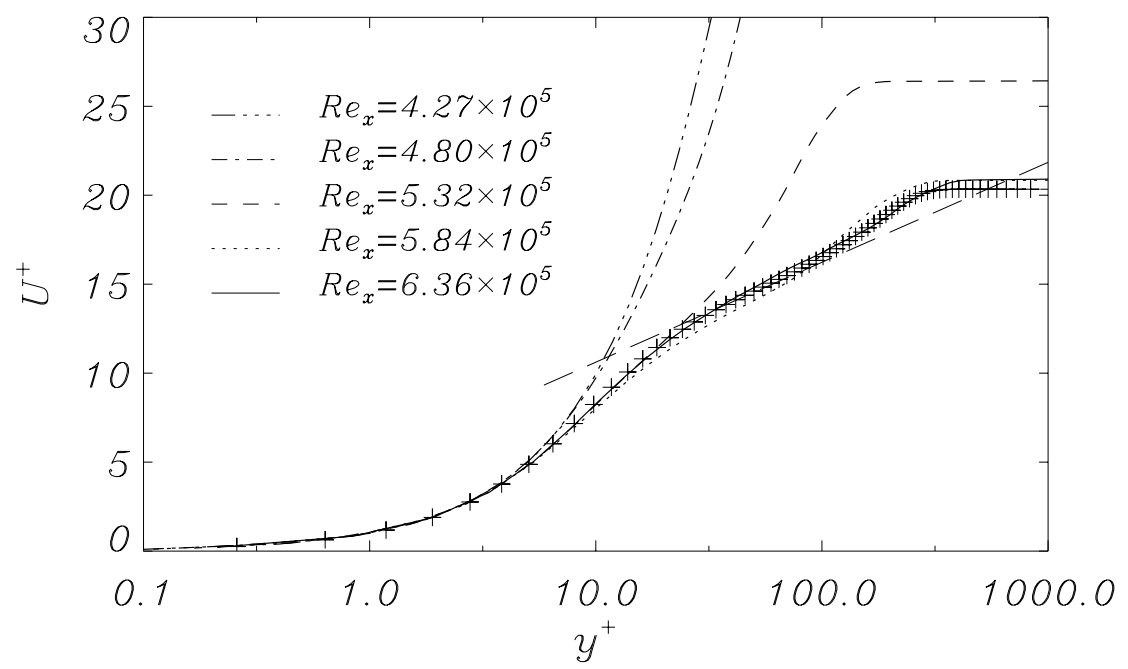

Figure 5: Mean streamwise velocity in wall units at various streamwise locations, + : DNS (Spalart, 1988); - - : $U^{+}=2.44 \ln y^{+}+5.0$; all other lines: LES.

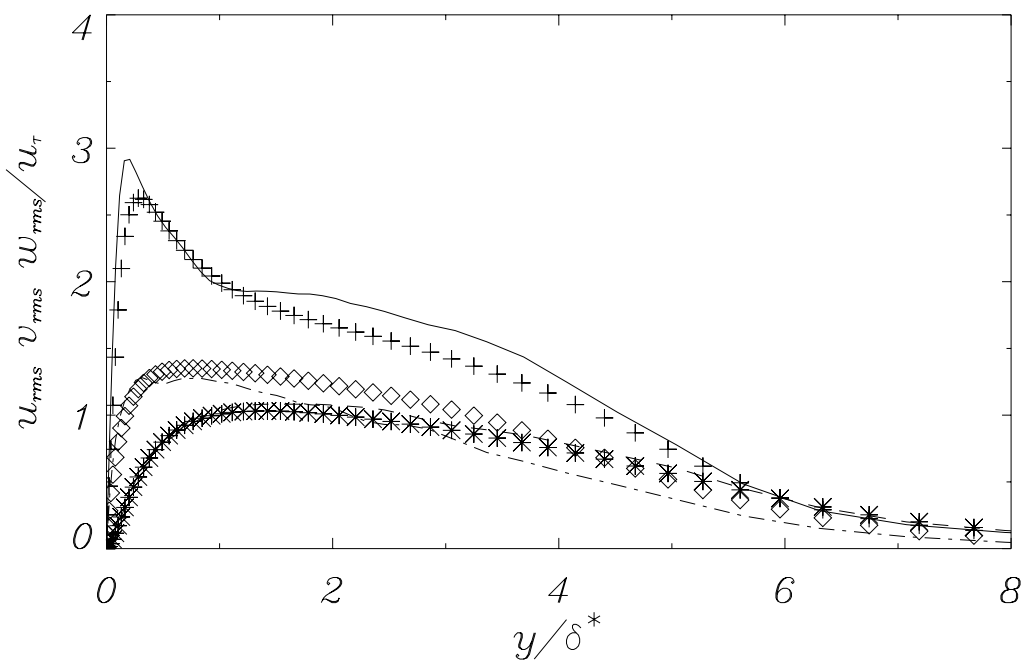

Figure 6: Wall-normal distribution of velocity $\mathrm{rms}$ at the last streamwise location, $R e_{x}=$ $6.36 \times 10^{5}$. Lines: LES, symbols: DNS. $-+: u_{r m s} ;---{ }^{*}: v_{r m s} ;--,, \diamond: w_{r m s}$. 


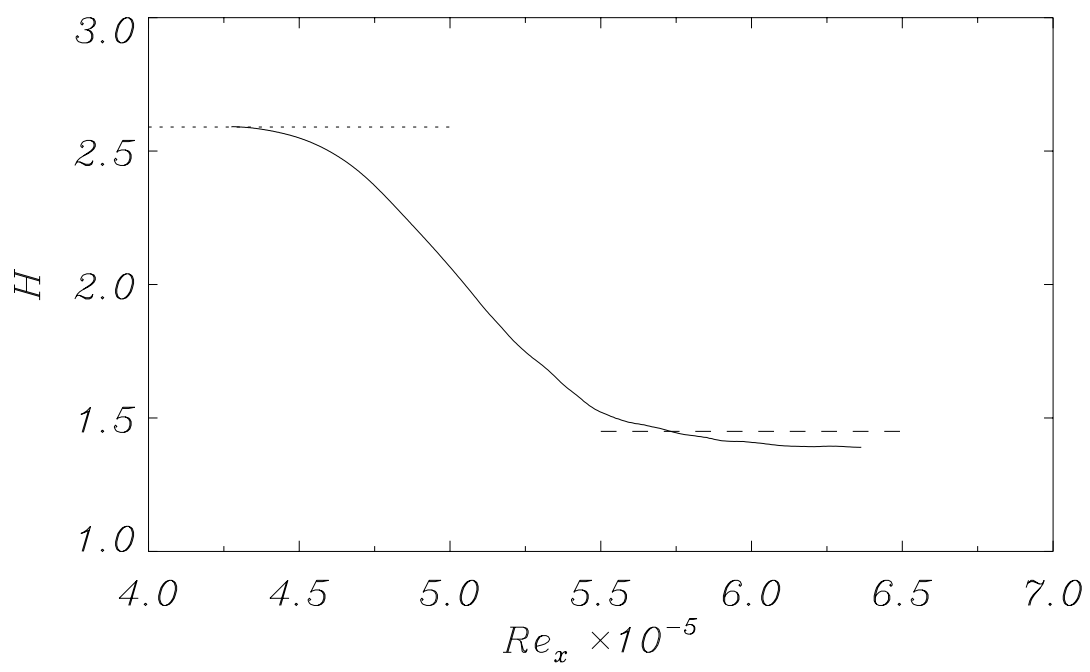

Figure 7: Streamwise variation of shape factor. —— : LES; ….... : laminar flow; --- : DNS

The local skin friction given in Figure 8 has the laminar value as long as the amplitudes of the disturbances are low, and begins to have a significant increase as the flow goes into laminar breakdown. The streamwise position where the skin friction begins to deviate from the laminar curve can be defined as the transition location, which in current calculation is $R e_{x} \approx 4.6 \times 10^{5}$. In the final stage of transition, the skin friction settles down to a level in agreement with the DNS (Spalart, 1988) of turbulent boundary layer at the corresponding Reynolds number $R e_{\delta^{*}}$. The turbulent skin friction, denoted by the chain-dot-dot-dot line, is given by the equation (see White, 1991),

$$
c_{f}=\frac{0.455}{\ln ^{2}\left(0.06 R e_{x}\right)} .
$$

with the virtual origin of the turbulent boundary layer moved from the leading edge towards the transition location. The turbulence skin friction thus obtained matches very well the result of LES.

\section{SGS model performance}

An important question for the simulation of transitional flows regards the capability of the LES to resolve the large vortical structures that arise during the nonlinear interaction stages of transition. In Fig. 9, the contours of the fluctuating streamwise velocity are shown in a horizontal plane located at $y=0.4\left(y^{+}=13\right.$ in the turbulent region) away from the wall. The staggered $\Lambda$-vortices typical of the subharmonic breakdown can be first observed at $x=640$ in the figure. They actually exist further upstream and by the time they are apparent in the figure, they have already become strong enough and gone into the later stage of development. The $\Lambda$-vortices are followed immediately by the laminar breakdown. Near the end of the computational box, the low speed streaks for the near-wall turbulent flow can be observed.

Figure 10 shows a time-sequence of contours of the vertical shear $\partial u^{\prime} / \partial y$ in the $z=0$ plane, where $u^{\prime}$ is the fluctuating velocity. A detached high shear layer first appears at $x=640$ in Fig. 10(a), which is also the location where the skin friction and the shape factor start to depart from their laminar values; a kink develops on the shear layer at $t=t_{o}+1.5 T$. Low pressure contour plots from the present simulation indicate a strong correspondence 


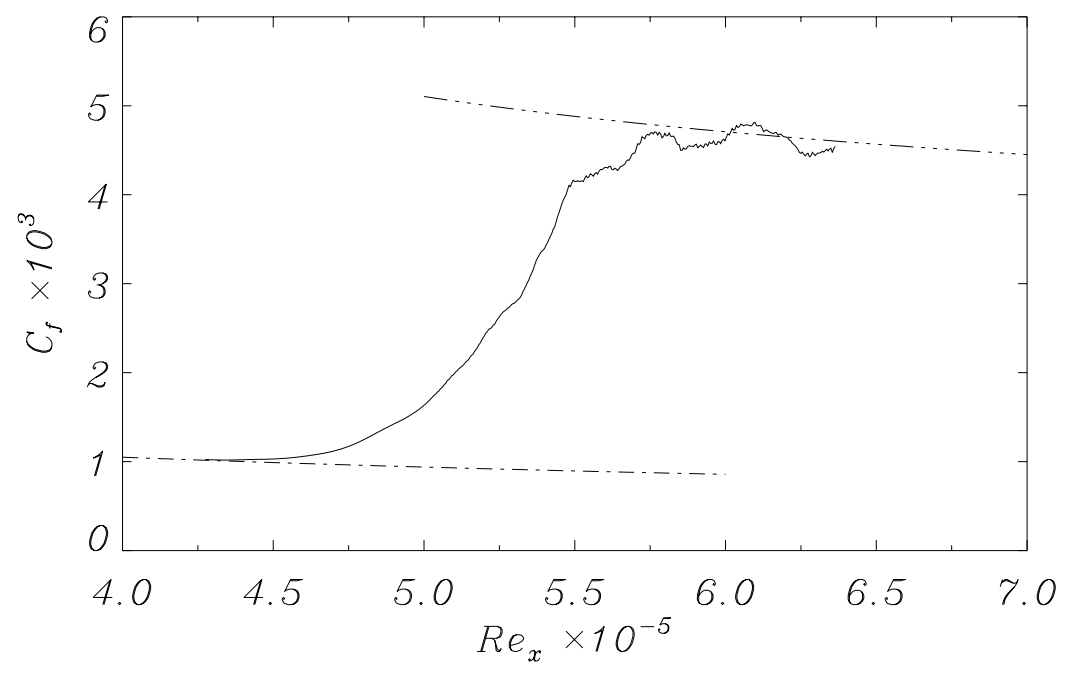

Figure 8: Streamwise variation of local skin friction. - : LES; - - : laminar flow $0.664 R e^{-0.5} ;-\cdots-$ : turbulent flow $0.455 / \ln ^{2}\left(0.06 R e_{x}\right)$ with the virtual origin at $R e_{x}=$ $2.9 \times 10^{5}$.

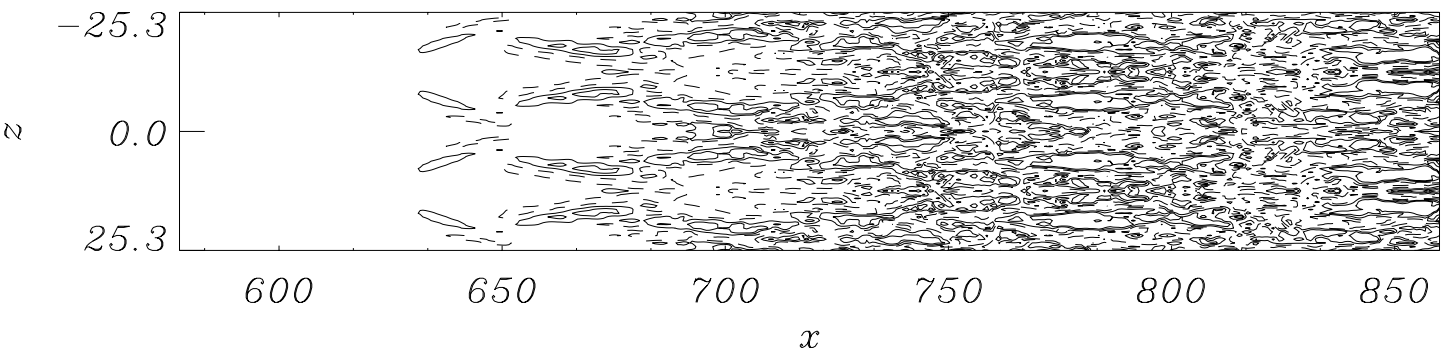

Figure 9: Contours of fluctuating streamwise velocity $u^{\prime}$ in the $x-z$ planes $y=0.38\left(y^{+}=13\right)$ at $t=t_{o}+1.5 T$, where $t_{o}=756$. The contour levels are between -0.15 and 0.15 . 


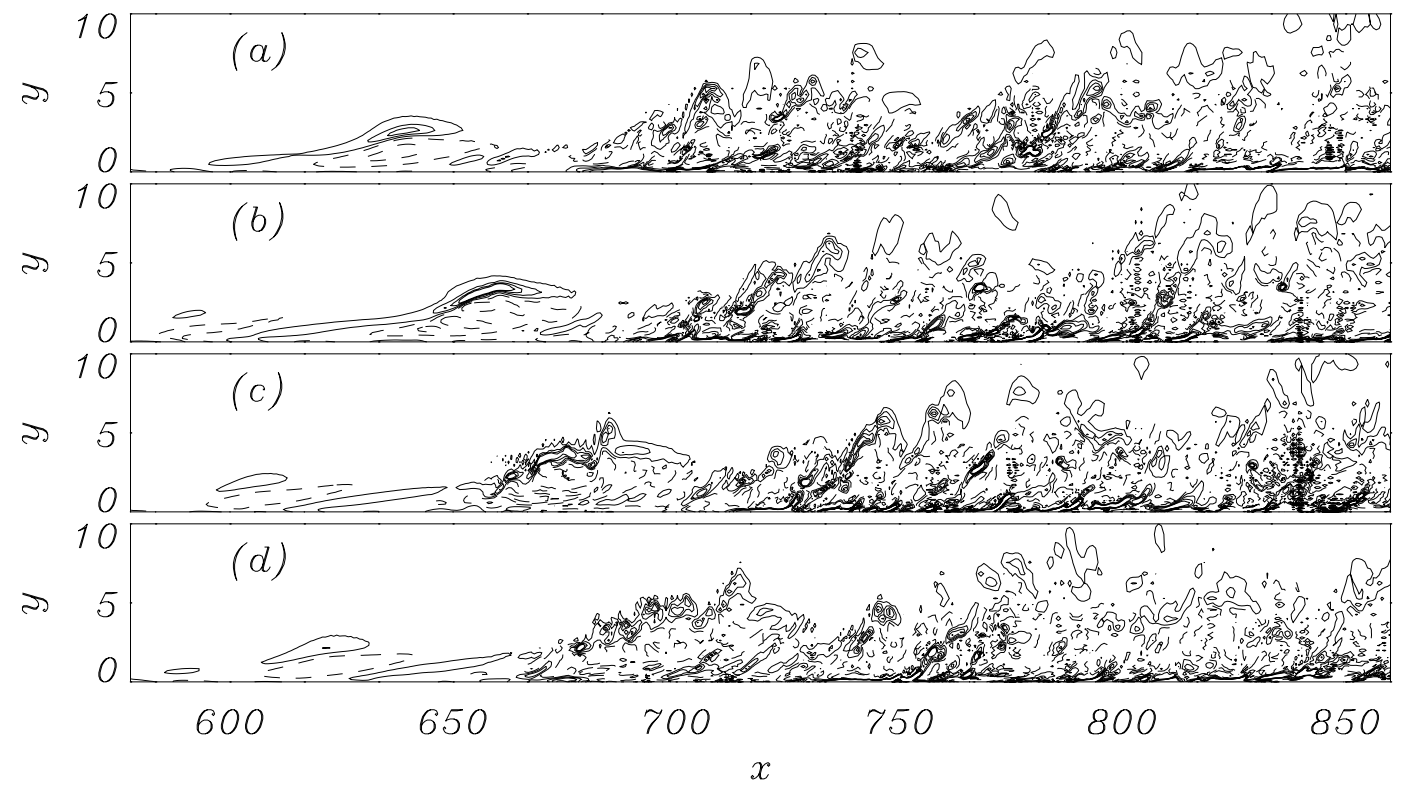

Figure 10: Time-sequence of vertical shear $\partial u^{\prime} / \partial y$ in a $x-y$ plane $(z=0)$. (a) $t=t_{o}+T$; (b) $t=t_{o}+1.5 T$; (c) $t=t_{o}+2 T$; (d) $t=t_{o}+2.5 T$, where $t_{o}=756$. The contour levels are between -0.45 and 0.45 .

between the kink in a shear layer and a vortex roll-up, as was also shown by Sandham and Kleiser (1992) by analyzing a temporal DNS database of the K-type breakdown. This small and energetic vortex can cause a spike in the velocity trace when it passes by the probe in an experiment. Multiple shear layer roll-ups occur in the subsequent development, and the flow finally reaches laminar breakdown and turbulence. In the turbulent region, the near-wall shear layers can be observed. It is worth pointing out that in the experiment of Kachanov and Levchenko (1984) no high-frequency spikes were observed, the transition featured a fast excitation of the broad spectrum of low-frequency fluctuations, including the subharmonic, followed by a filling of spectrum by an interaction of low-frequency fluctuations with the fundamental wave and its harmonics.

In the present simulation, the $\Lambda$-vortices, the high shear layers and their roll-ups, and the streak structures are all captured, even though the grid resolution is marginal during the laminar breakdown, as indicated by the low level oscillations in the contours of Fig. 10. It can be concluded that even with much coarser grid than in DNS, LES using the localized dynamic model can predict transition accurately not only in terms of statistics, but even as far as the local behavior is concerned.

One of the advantages of the dynamic model used in this calculation is its ability to adjust the model coefficient in time according to the flow conditions. By using the localized version of the dynamic model, the spatial variation of the flow can also be included. In the first box of the present simulation, the dynamic model gives essentially zero eddy viscosity because the transition is still in its early stages; this results in the correct prediction of the spatial growth of the disturbances. Figure 11 shows the wall-normal distribution of the mean eddy viscosity at the five evenly spaced streamwise locations shown in Fig. 5. At the first two streamwise locations, the eddy viscosity is essentially zero because the small scale disturbances are still not measurable in the flow; it becomes significant starting from the third location where 


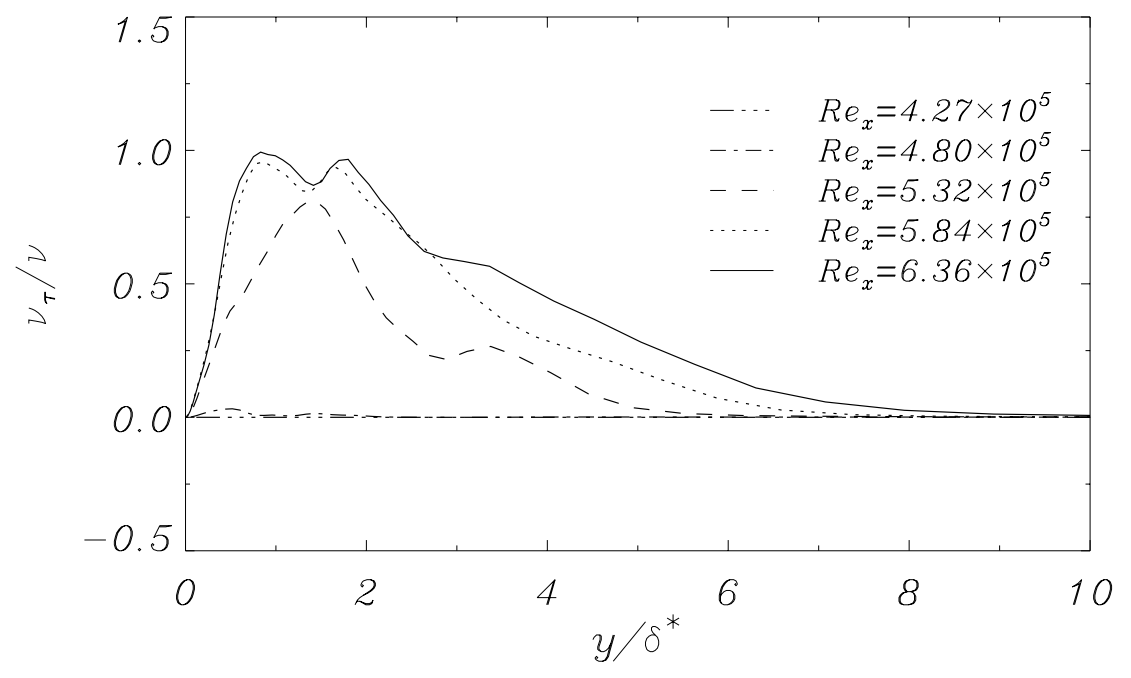

Figure 11: Mean eddy viscosity distributions at various streamwise locations.

the laminar breakdown occurs; in the later stages of transition and in the turbulent stage it reaches the same magnitude as the molecular viscosity within the boundary layer.

Figure 12 shows a time-sequence of contours of the SGS eddy viscosity in the $z=0$ plane. Comparing this figure with the corresponding Fig. 10 reveals again that the dynamic model only turns on the eddy viscosity in the transition region and afterwards, where small scales are present in the flow. Some negative values of eddy viscosity indicate backscatter (energy transfer from small scales to large scales) in the transition and the turbulent regions. Moreover, the figure suggests that the eddy viscosity is very small in the first spike stage, and becomes significant only during and after the multi-spike stage. This is clearly shown in Fig. 13 by the time-series of the disturbance velocity $u$ and the SGS eddy viscosity $\nu_{t}$ at four locations in the flow field, whose positions were marked by the bullets in Figure 12a. In the laminar region where the disturbance is still small, the eddy viscosity given by the localized dynamic model is essentially zero (Fig. 13a). The first spike stage is indicated by a strong perturbation in the velocity trace at around $t_{0}+2.5 T$, but the eddy viscosity remains small compared to the molecular one (Fig. 13b). In the multi-spike stage (Fig. 13c), the eddy viscosity has significant values at the time when multiple spikes appear in the velocity trace, because of the emergence of small scales; it, however, remains small before and after the spikes. Figure 13d shows the chaotic variation of the velocity and the eddy viscosity in the turbulent region.

It has been shown that the localized SGS model used in the present study has the ability to adjust itself locally both in space and time according to the characteristics of the flow field. This is essential to the large-eddy simulation of transitional flows, since intermittency is a dominant phenomenon during transition. A SGS model that lacked this capability could result in over-damping of the disturbances and incorrect prediction of the transition process, as does the original Smagorinsky model. In addition, the eddy viscosity given by the localized model is zero in the first spike stage, and only multiple spikes can give rise to non-zero values of eddy viscosity. In a direct numerical simulation of transition, the grid resolution has to be 


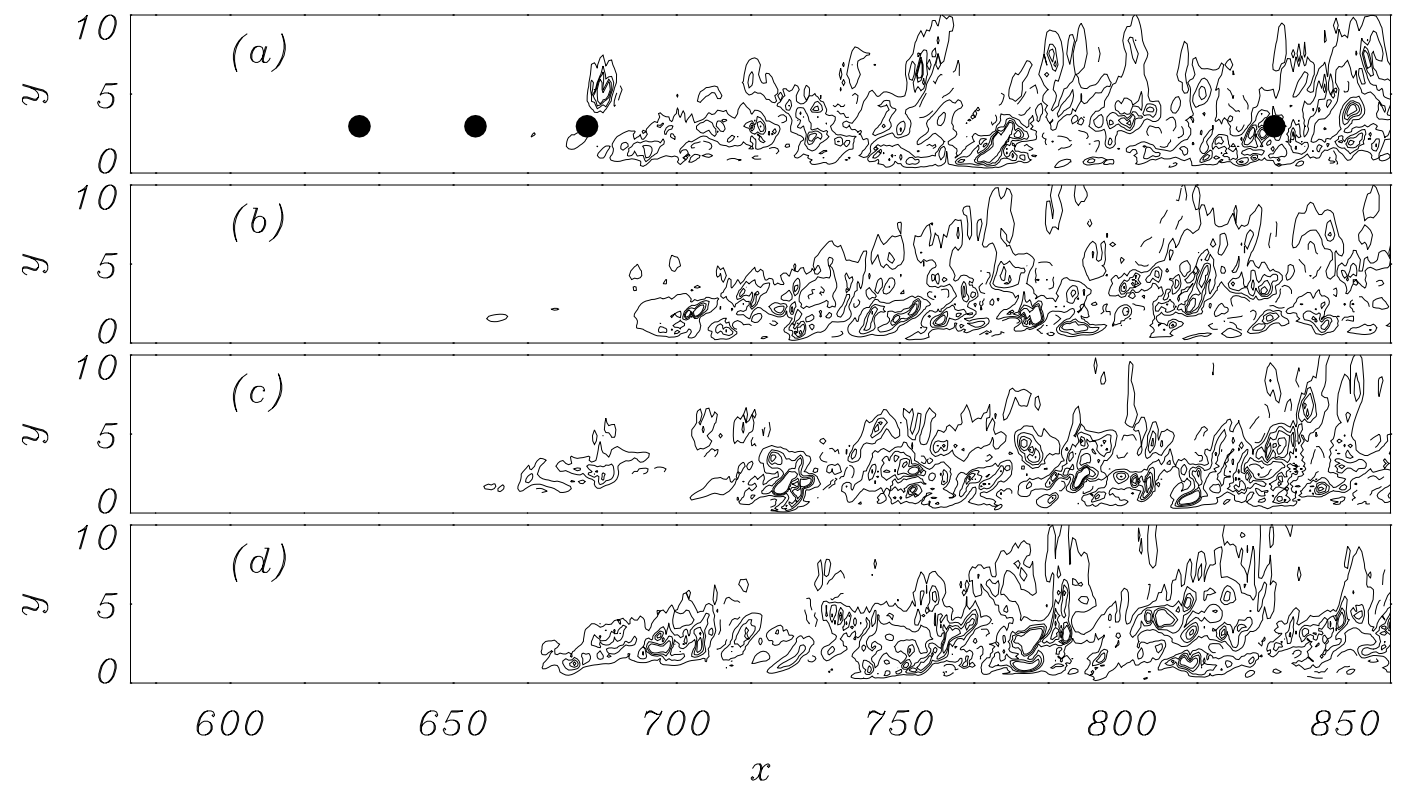

Figure 12: Time-sequence of the eddy viscosity in the $(z=0)$ plane. (a) $t=t_{o}+T$; (b) $t=t_{o}+1.5 T$; (c) $t=t_{o}+2 T$; (d) $t=t_{o}+2.5 T$. The contour levels are between -1 and 3 .

increased before the multiple spike stage, since the effect of the small scale disturbances on the large ones then becomes significant and cannot be neglected.

Also shown in Fig. 13 are the eddy viscosities obtained from simulations using the Smagorinsky model with the intermittency modification (Piomelli et al. 1990)

$$
\nu_{T}=\left(\frac{H_{l}-H}{H_{l}-H_{t}}\right)^{2}\left(\Delta c_{s}\right)^{2} \sqrt{2 s_{i j} s_{i j}}
$$

where $H$ is the shape factor, and the low-Reynolds-number model (Voke, 1995)

$$
\nu_{T}=\nu_{s}-(\nu / \beta)\left[1-\exp \left(-\beta \nu_{s} / \nu\right)\right]
$$

where $\nu_{s}$ is given by the original Smagorinsky model, $c_{s}$ is chosen as 0.1 , and $\beta=4.5$. According to Voke (1996), only the fluctuating strain rate $s_{i j}$, rather than the total strain, should be used to compute $\nu_{s}$. The van Driest wall damping function is used for both models. The calculations start from the same flow field of LES at $t=t_{o}$. It can be observed clearly that the eddy viscosity given by the low-Reynolds-number model is also insignificant in the laminar region, whereas in the first spike stage, the model gives much higher eddy viscosity; its variation closely resembles that of the vertical shear, reflecting the fact that $\partial u / \partial y$ is the dominant term in the strain-rate tensor; the spike is thus weakened. The higher viscosity of the low-Reynolds-number model also results in the smearing out of the multiple spikes in Fig. 13c. On the other hand, the Smagorinsky model with the intermittency modification gives results in agreement with the dynamic model well into the one-spike stage; it, however, gives smaller eddy viscosity in the multi-spike stage due to the intermittency attenuation. 
(a)

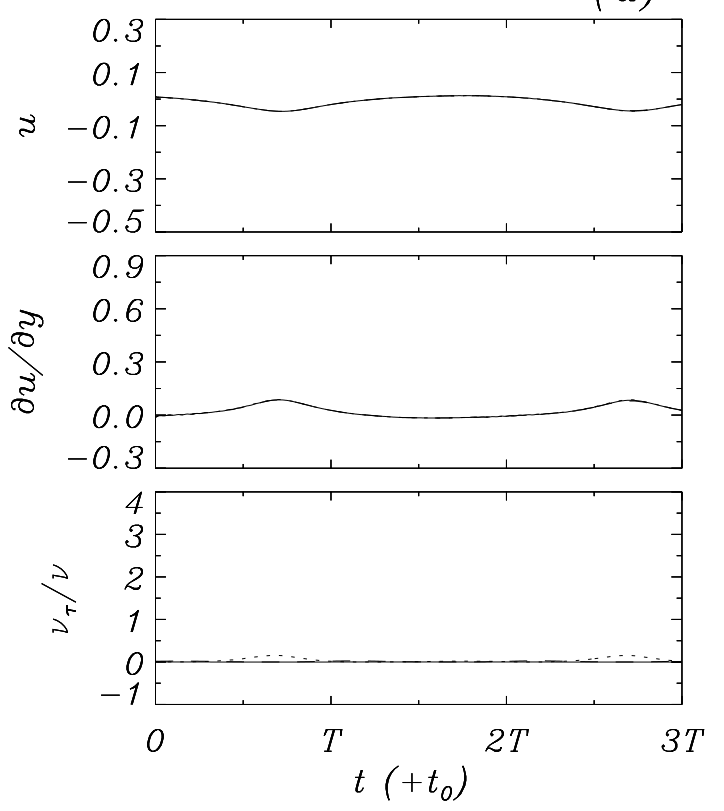

(c)
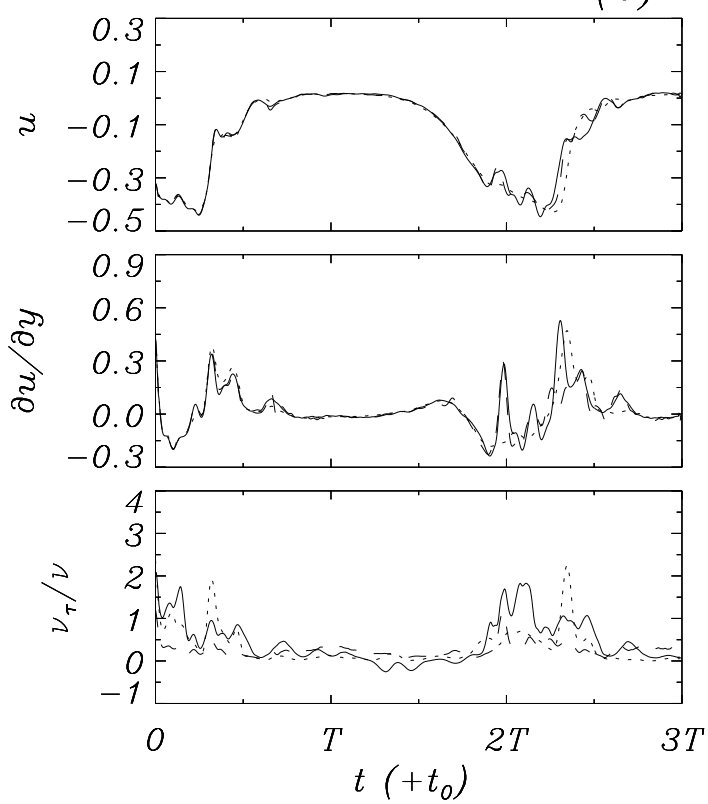

(b)
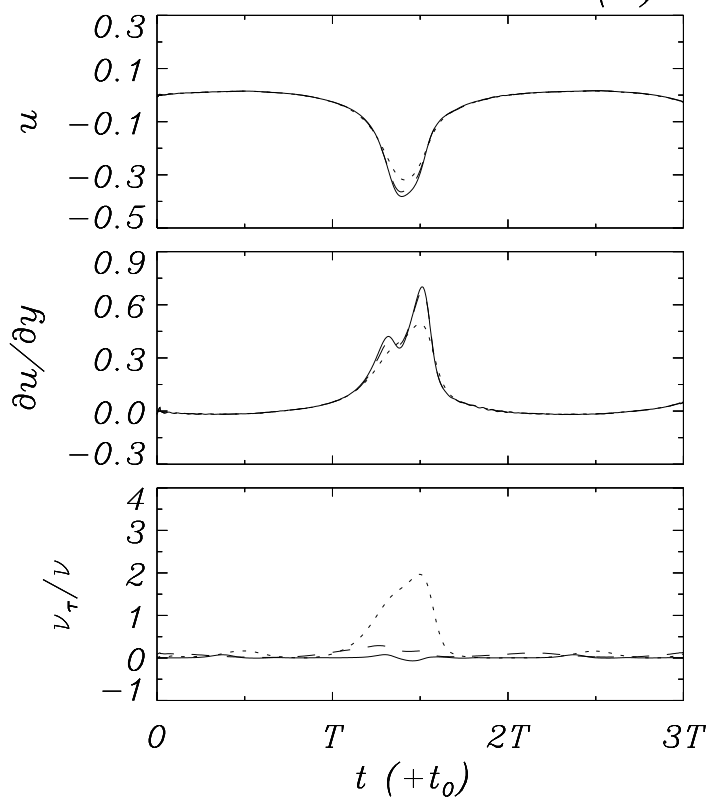

(d)
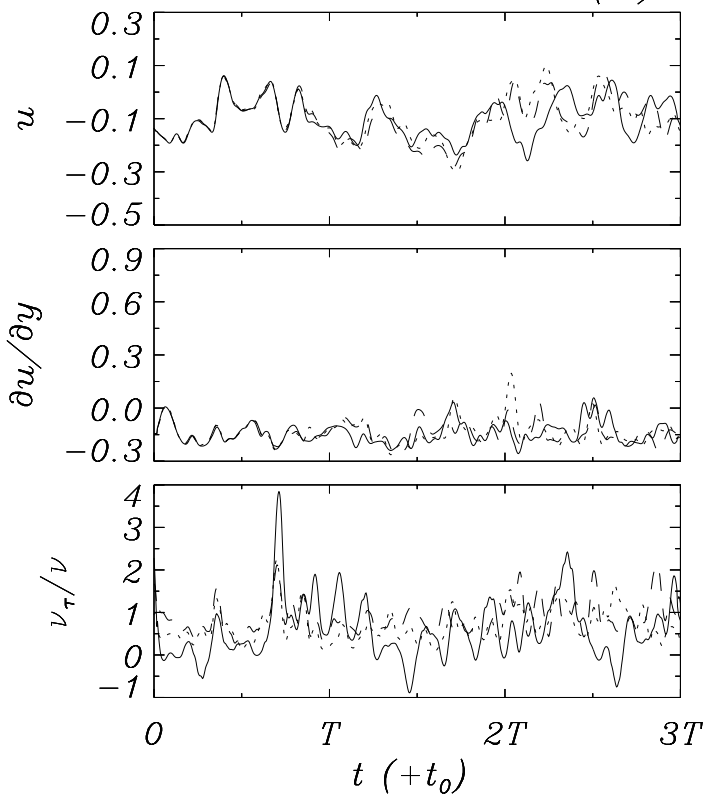

Figure 13: Time history of the disturbance velocity $u$, the vertical shear $\partial u / \partial y$ and the eddy viscosity $\nu_{t}$ at $y=3.0, z=0$ and (a) $x=629$; (b) $x=655$; (c) $x=680$; (d) $x=834$. The eddy viscosities are given by: — : localized dynamic model; $\cdots \cdots \cdots \cdot$ : low-Reynolds-number Smagorinsky model; --- : Smagorinsky model with the intermittency modification. 


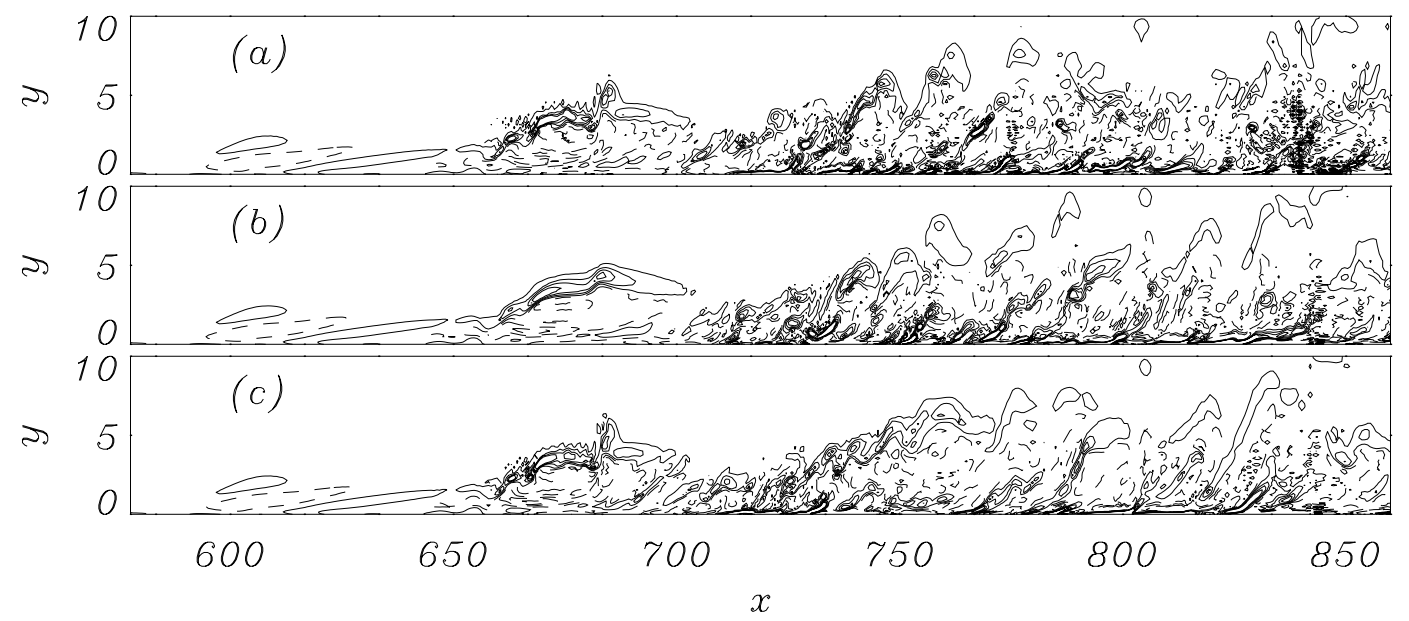

Figure 14: Time-sequence of vertical shear $\partial u^{\prime} / \partial y$ in a $x-y$ plane $(z=0)$ and at $t=t_{o}+2 T$. (a) Localized dynamic model; (b) low-Reynolds-number Smagorinsky model; (c) Smagorinsky model with the intermittency modification. The contour levels are between -0.45 and 0.45 .

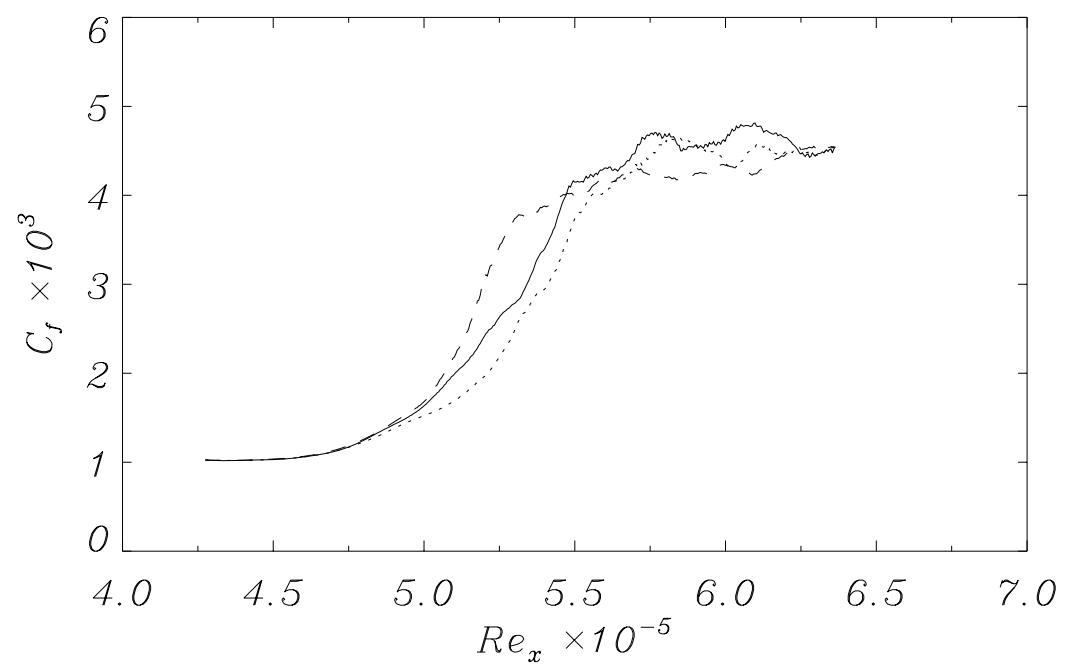

Figure 15: Streamwise variation of skin friction. —— : Localized dynamic model; ….... : low-Reynolds number Smagorinsky model; --- : Smagorinsky model with the intermittency modification. 
In the turbulent region, both models predict positive eddy viscosity and its peak values are smaller.

Figure 14 compares the contours of $\partial u^{\prime} / \partial y$ for different SGS models. The shear layer roll-up at $x=680$ in the low-Reynolds-number model simulation is clearly not as strong as in other two cases, due to excessive damping provided by the eddy viscosity in the first spike stage; this also causes the transition to occur somewhat later and the increase in $c_{f}$ to be less steep, as shown in Fig. 15. The modified Smagorinsky model gives higher skin friction in the laminar breakdown region because of the attenuation of the SGS eddy viscosity by intermittency. The scattering of the data points in the turbulent region on Fig. 15 is due to insufficient averaging. The comparisons here between three models tends to indicate that the localized dynamic model does a better job, because the higher shear layer it resolves is sharper and the mean velocity profile it gives compares with DNS better than those of the other two. Furthermore, the localized dynamic model has no adjustable constant or ad hoc parameter. However, this issue need to be further investigated, a comparison with DNS is desired.

\section{Conclusions}

Large-eddy simulation of subharmonic type transition in a flat plate boundary layer was carried out. It was observed that the simulation predicts accurately the development of disturbances in the early stages, the shear layers and vortical structures in the laminar breakdown stage, and the turbulent statistics in the turbulent stage. In addition, it has been shown that the skin friction for the simulation during laminar breakdown falls below the value of natural transition. The localized dynamic SGS model has the desired capability to adjust itself locally both in space and time according to the characteristics of the flow field. It yields very small eddy viscosity in the laminar as well as the one spike stage, but gives significant values in the multi-spike and the turbulent stages, due to the emergence of energy in small scales of motion. The study also shows that the intermittency SGS model resembles the behavior of the localized dynamic model closely in the first-spike stage of transition, however, it gives smaller eddy viscosity in the multiple-spike stage; the low-Reynolds-number model predicts large values of eddy viscosity even in the first-spike stage.

\section{Acknowledgments}

The authors would like to thank Dr. Peter Voke for helpful discussions. 


\section{References}

Bestek, H., Kloker, M. and Müller, W., 1994 "Spatial direct numerical simulation of boundary layer transition under strong adverse pressure gradient," AGARD-CP-551, 32-1.

Chorin, A. J., 1968 "Numerical solution of the Navier-Stokes equations," Math. Comput. 22, 745/

Corke, T. C. and Mangano, R. A., 1989 "Resonant growth of three-dimensional modes in transition Blasius boundary layers," J. Fluid Mech. 209, 93.

Crouch, J. D. and Herbert, T., 1993 "Nonlinear evolution of secondary instabilities in boundary-layer transition," Theoret. Comput. Fluid Dyn. 4, 151.

Ducros, F., Comte, P., and Lesieur, M., 1996 "Large-eddy simulation of transition to turbulence in a boundary layer developing spatially over a flat plate." J. Fluid Mech. 326, 1 .

Fasel, H. F., Rist, U. and Konzelmann, U., 1990 "Numerical investigation of the threedimensional development in boundary-layer transition," AIAA J. 28, 1.

Germano, M., Piomelli, U., Moin, P., and Cabot, W.H., 1991 "A dynamic subgrid-scale eddy viscosity model," Phys. Fluids A 3, 1760.

Herbert, T., 1988 "Secondary instability of boundary layers," Ann. Rev. Fluid Mech. 20, 487.

Joslin, R. D., Streett, C. L. and Changa, C.-L., 1992 "3-D incompressible spatial direct numerical simulation code validation study - A comparison with linear stability and parabolic stability equation theories for boundary-layer transition on a flat plate," NASA TP-3205.

Joslin, R. D., Streett, C. L. and Chang, C.-L., 1993 "Spatial Direct Numerical Simulation of Boundary-Layer Transition Mechanisms: Validation of PSE Theory," Theoretical and Computational Fluid Dynamics 4, 271.

Joslin, R.D. and Streett, C.L., 1994 "The Role of Stationary Crossflow Vortices in BoundaryLayer Transition on Swept Wings," Phys. Fluids 6, 3442.

Joslin, R.D., 1995 "Evolution of Stationary Crossflow Vortices in Boundary Layers on Swept Wings," AIAA Journal 33, 1279.

Joslin, R.D., 1995 "Direct Simulation of Evolution and Control of Three-Dimensional Instabilities in Attachment-Line Boundary Layers," J. Fluid Mech. 291, 369.

Kachanov, Y. S. and Levchenko, V. Y., 1984 "The resonant interaction of disturbances at laminar-turbulent transition in a boundary layer," J. Fluid Mech. 138, 209.

Kachanov, Y. S., 1994 "Physical mechanisms of laminar-boundary-layer transition," Ann. Rev. Fluid Mech. 26, 411

Klebanoff, P. S., Tidstrom, K. D. and Sargent, L. M., 1962 'The three-dimensional nature of boundary layer instability," J. Fluid Mech. 187, 61. 
Kleiser, L. and Zang, T. A., 1991 "Numerical simulation of transition in wall-bounded shear flows," Ann. Rev. Fluid Mech. 12, 1.

Lundbladh, A., Schmid, P. J., Berlin, S. and Henningson, D. S., 1994 "Simulations of bypass transition for spatially evolving disturbances," AGARD-CP-551, 18-1.

Murdock, J. W., 1986 "Three-dimensional numerical study of boundary-layer instability," AIAA Paper No. 86-0434.

Piomelli, U., Zang, T. A., Speziale, C. G. and Hussaini, M. Y., 1990 "On the large eddy simulation of transitional wall-bounded flows," Phys. Fluids A 2, 257.

Piomelli, U. and Zang, T. A., 1991 "Large-eddy simulation of transitional channel flow" Computer Phys. Comm. 65, 224.

Piomelli, U. and Liu, J., 1995 "Large eddy simulation of rotating channel flows using a localized dynamic model." Phys. Fluids 7, 839.

Roach, P. E. and Brierley, D. H., 1992 "The influence of a turbulent free-stream on zero pressure gradient transitional boundary layer development," In Numerical Simulation of Unsteady Flows and Transition to Turbulence, edited by Pironneau, O., Rodi, W., Rhyming, I. L., Savill, A. M. and Truong, T. V., Cambridge University Press, New York, 398.

Sandham, N. D. and Kleiser, L., 1992 "The late stages of transition to turbulence in channel flow," J. Fluid Mech. 245, 319.

Smagorinsky, J., 1963 "General circulation experiments with the primitive equations. I. The Basic experiment," Monthly Weather Review 91, 99.

Spalart, P. R., 1988 "Direct simulation of a turbulent boundary layer up to $R_{\theta}=1410$," $J$. Fluid Mech. 187, 61.

Streett, C. L., and Macaraeg, M. G., 1989 "Spectral multi-domain for large-scale fluid dynamics simulations," Int. J. Applied Num. Math. 6, 123.

Voke, P. R., and Yang, Z., 1995 "Numerical study of bypass transition," Phys. Fluids 7, 2256 .

Voke, P. R., 1996 Private communication.

White, M. W., 1991 Viscous Fluid Flow, 2nd edition, McGraw-Hill. 